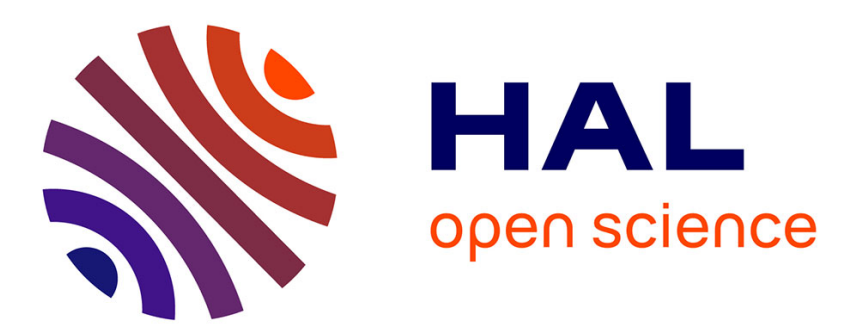

\title{
Vous avez dit démocratie? L'extension des droits de citoyen en Océanie Française. Enjeux, pratiques et limites
}

Isabelle Merle

\section{- To cite this version:}

Isabelle Merle. Vous avez dit démocratie? L'extension des droits de citoyen en Océanie Française. Enjeux, pratiques et limites. Outre-Mers Revue d'Histoire, 2019, Quels citoyens pour l'empire?, 404-405, pp.17 - 40. 10.3917/om.192.0017 . hal-03524747

\section{HAL Id: hal-03524747 \\ https://hal.science/hal-03524747}

Submitted on 31 Jan 2022

HAL is a multi-disciplinary open access archive for the deposit and dissemination of scientific research documents, whether they are published or not. The documents may come from teaching and research institutions in France or abroad, or from public or private research centers.
L'archive ouverte pluridisciplinaire HAL, est destinée au dépôt et à la diffusion de documents scientifiques de niveau recherche, publiés ou non, émanant des établissements d'enseignement et de recherche français ou étrangers, des laboratoires publics ou privés. 


\title{
VOUS AVEZ DIT DÉMOCRATIE ? L'EXTENSION DES DROITS DE CITOYEN EN OCÉANIE FRANÇAISE. ENJEUX, PRATIQUES ET LIMITES
}

\author{
$\underline{\text { Isabelle Merle }}$
}

Société Française d'Histoire des Outre-Mers (S.F.H.O.M) | « Outre-Mers »

2019/2 N 404-405 | pages 17 à 40

ISSN 1631-0438

DOI 10.3917/om.192.0017

Article disponible en ligne à l'adresse :

https://www.cairn.info/revue-outre-mers-2019-2-page-17.htm

Distribution électronique Cairn.info pour Société Française d'Histoire des Outre-Mers (S.F.H.O.M).

(C) Société Française d'Histoire des Outre-Mers (S.F.H.O.M). Tous droits réservés pour tous pays.

La reproduction ou représentation de cet article, notamment par photocopie, n'est autorisée que dans les limites des conditions générales d'utilisation du site ou, le cas échéant, des conditions générales de la licence souscrite par votre établissement. Toute autre reproduction ou représentation, en tout ou partie, sous quelque forme et de quelque manière que ce soit, est interdite sauf accord préalable et écrit de l'éditeur, en dehors des cas prévus par la législation en vigueur en France. Il est précisé que son stockage dans une base de données est également interdit. 


\title{
Vous avez dit démocratie? \\ L'extension des droits de citoyen en Océanie Française. Enjeux, pratiques et limites
}

Isabelle MERLE *

\begin{abstract}
Résumé
Cet article vise à interroger les politiques qui furent conjointement menées par la France à l'égard des populations de l'Océanie, nouvellement conquises, entre 1840 et I880. Ces politiques opèrent des différentiations en fonction des populations rencontrées, Tahitiens, Marquisiens, Kanaks, $\mathrm{du}$ fait du contexte dans lequel elles se trouvent, des caractéristiques " raciales" que les officiels français leur attribuent ou encore des rapports de force instaurés localement avec les chefferies et les nouveaux compétiteurs européens en voie d'installation dans la région. Dans ce contexte compliqué, les Tahitiens se voient accorder la citoyenneté française en I880, tandis que les Kanak sont placés sous le statut de Français non citoyens comme la grande majorité des indigènes de l'Empire colonial et par voie de conséquence le régime répressif de l'indigénat.
\end{abstract}

Mots-clés : Colonisation, Pacifique, Citoyenneté, Indigène, NouvelleCalédonie, Tahiti.

\begin{abstract}
This article questions the policies that were jointly pursued by France towards the populations of newly conquered Oceania, between I840 and I880. These policies differentiated the populations encountered, Tahitians, Marquesans, Kanak, acording to the context in which they were found, the "racial" characteristics that French officials attributed to them, and the power relations established locally with the chiefdoms and the new European competitors in the process of settling in the region. In this complicated context, the Tahitians were granted French citizenship in I880, while
\end{abstract}

* Isabelle Merle est directrice de recherche au CNRS (Aix Marseille Université, CNRS, EHESS, CREDO, UMR 7308, Marseille, France), historienne et spécialiste de l'histoire de la colonisation et en particulier de l'histoire du Pacifique. Elle vient de publier avec Adrian Muckle, L'Indigénat. Genèses dans l'Empire français. Pratiques en Nouvelle-Calédonie, CNRS Éditions, Paris, 2019. 
the Kanak were granted the status of French non-citizens, and consequently, subjected to the repressive regime of the indigénat just as the vast majority of the indigenous peoples of the French Empire.

Keywords: Colonisation, Pacific, Citizenship, Indigenous, New Caledonia, Tahiti.

En I927, le célèbre juriste Henry Solus, auteur du Traité de la condition des indigènes en droit privé, écrit, à propos des « indigènes de Tahiti et dépendances ": "À la suite de la cession faite à la France par le roi Pomaré $\mathrm{V}$ de ses États (Tahiti et dépendances), la loi du 30 décembre I880, décida en son article $3:$ La nationalité française est acquise de plein droit à tous les anciens sujets du Roi ». Et Solus de poursuivre : "Cette formule, sur le sens de laquelle on pourrait éprouver des doutes puisqu'elle pourrait aussi bien convenir à des indigènes qui ne sont que sujets français et non citoyens français, perd néanmoins toute ambiguité quand on consulte le rapport de Mr Godin ". Ce dernier, rapporteur à la Chambre des députés, lors de la séance du I8 décembre I880, confirme que la commission chargée du projet de loi portant sur la transformation du protectorat en colonie a reconnu que « les habitants de Tahiti sont français et jouissent par suite de tous les droits des citoyens français " I. D'où la conclusion qu'en tire Solus : "Il semble bien que les habitants de Tahiti soient citoyens français " ${ }^{2}$.

On sent poindre ici une nette hésitation car, en I927, Solus a du mal à préciser en quoi les Tahitiens sont effectivement citoyens alors qu'ils ne votent plus pour le Conseil colonial du territoire depuis I903 et qu'ils sont largement perçus comme des indigènes au même titre que les sujets de l'Empire. Comme d'autres, il peine à se souvenir que les sujets du Royaume de Pomaré $\mathrm{V}$ ont accédé pendant un temps à l'exercice de la citoyenneté active entre I88I et I903.

La césure entre " citoyens " et "non citoyens " dans les colonies a été définie, on le sait, par le Sénatus-consulte promulgué le I4 juillet I 865 en Algérie qui, traitant du statut des personnes indigènes, confirmait d'un côté leur appartenance à la communauté nationale (la qualité de Français), mais affirmait d'un autre côté leur non appartenance à la communauté des citoyens du fait du maintien de leur statut personnel supposé incompatible avec le code civil (sauf renoncement à ce statut personnel et soumission aux contraintes d'une demande de naturalisation individuelle). Ce régime "d'indigène français non citoyen " a été

I. Rapport Godin, cité dans le Recueil général des lois et des arrêts : en matière civile, criminelle, commerciale et de droit public de J.-B. Sirey, I88I, p. 86, note 2. Source Gallica. bnf.fr.

2. Henry Solus, Traité de la condition des indigènes en droit privé, Paris, Société anonyme du Recueil Sirey, 1927, p. 33. 
largement étendu dans l'Empire français pour devenir la norme dans la première partie du $\mathrm{xx}^{\mathrm{e}}$ siècle 3 .

Il comportait dès l'origine des exceptions, la citoyenneté active ayant été accordée, dans la foulée de l'abolition de l'esclavage à partir de I848, à l'ensemble des hommes majeurs, y compris les esclaves nouvellement affranchis, des anciennes colonies (Martinique, Guadeloupe, Guyane, Réunion) ainsi qu'aux anciens sujets du Roi des comptoirs français (les Quatre communes du Sénégal et les Établissements français de l'Inde). Pour l'auteur de la loi, Victor Schœlcher, la longue histoire du lien entre ces territoires et la France justifiait pleinement l'octroi de la citoyenneté à leurs habitants 4 . L'octroi de la citoyenneté aux anciens sujets du Royaume de Pomaré s'inscrit dans une histoire très différente et constitue en cela une nouvelle exception à la norme impériale de l'indigène français non citoyen.

Tahiti et ses dépendances font partie de la seconde vague de l'expansion française qui s'ouvre avec la conquête de l'Algérie à partir de I830 et constituent, avec les Marquises conquises en I842 et la NouvelleCalédonie en I853, les maillons d'un empire français en expansion en Océanie auxquels viennent s'adjoindre le protectorat de Wallis et Futuna en I844, les Gambier et les îles Sous-le-Vent, respectivement annexées en I88I et en I887. Alors que le statut de citoyen est clairement refusé aux "nouveaux entrants " dans ce second empire colonial français en voie de formation, Algériens, Kanaks, Marquisiens puis Cochinchinois et ceux qui suivront, il est accordé en I880, aux sujets du Roi Pomaré. La conséquence majeure de cette distinction est la suivante : les premiers, Français non citoyens, vont connaitre l'application du régime de l'indigénat tout au long de la période coloniale tandis que les seconds, accédant au statut de citoyens, vont y échapper, tout au moins dans le principe 5 .

3. Laure Blévis, "Sociologie d'un droit colonial : citoyenneté et nationalité en Algérie (I865-1947. Une exception républicaine ? ", thèse de doctorat, Aix-Marseille université, 2004 ; Id., "L'Invention de l'indigène, Français non citoyen ", dans Abderrahmane Bouchène, Jean-Pierre Peyroulou, Ounassa Siari-Tengour et Sylvie Thénault (dir.), Histoire de l'Algérie à la période coloniale 1830-1962, Paris, La Découverte, 2014, p. 212-218; Yerri Urban, L'Indigène dans le droit colonial français, I865-1955, Clermont-Ferrand, Fondation Varenne, 20I I.

4. Sylviane Larcher, L'Autre citoyen, Paris, Armand Colin, 20I4. Damien Deschamps, "La République aux Colonies : le citoyen, l'indigène et le fonctionnaire : citoyenneté, cens civique et représentation des personnes, le cas des Établissements français de l'Inde et la genèse de la politique d'association (vers I848-vers I900) ", thèse de doctorat, Université de Grenoble 2, I998.

5. Le régime de l'indigénat, en effet, n'est pas appliqué aux Antilles, à Pondichéry ou à Tahiti. Ceci n'empêche pas des formes pratiques de contrôles, de corvées ou d'impôts, qui se rapprochent des réglementations de l'indigénat. Il faut noter qu'il est appliqué formellement aux Îles Sous-le-Vent (Polynésie) entre I897 et 1907 en punition de la résistance dont les habitants, évangélisés par les protestants britanniques comme leurs voisins de l'île au vent (Tahiti), ont fait preuve contre l'intrusion française et la prise de possession. Sur la question du régime de l'indigénat et de ses extensions impériales : Isabelle Merle et Adrian Muckle, L'Indigénat. Genèses dans l'empire français. Pratiques en Nouvelle-Calédonie, Paris, CNRS Editions, 2019, en particulier p. II8-120; 
Les historiens, spécialistes de l'histoire de la Polynésie, ont cherché quelques explications à cette bizarrerie que constitue l'exception citoyenne tahitienne. Les uns invoquent la longue influence des missionnaires britanniques et protestants et leur emprise préalable à l'installation française sur les chefferies tahitiennes ${ }^{6}$. D'autres insistent sur "les logiques d'assimilation " propres au protectorat français à Tahiti, d'autres encore parlent d'un don en reconnaissance d'une allégeance à la France des élites tahitiennes 7. La question est balayée relativement rapidement comme si elle s'imposait comme une évidence. La nature et l'ancienneté des liens tissés avec la France par les Tahitiens expliqueraient naturellement l'accès qui leur est octroyé en $\mathrm{I} 880$ à la citoyenneté française, tandis que les Kanak et par extension les Marquisiens, catalogués comme "primitifs ", ne pouvaient être traités différemment de la masse des indigènes non citoyens de l'Empire, condamnés à subir le régime de l'indigénat. La Nouvelle-Calédonie ou les Marquises, pour les spécialistes de l'histoire de Tahiti, connaissent l'expérience commune des colonisés français à laquelle les Tahitiens auraient échappé par la condition particulière qui leur est faite en I880.

Nous voudrions, dans cet article, interroger ces évidences en prenant tout d'abord le recul nécessaire sur les politiques qui furent conjointement menées par la France à l'égard des populations océaniennes nouvellement conquises entre I840 et I880, dans ce qui deviendra l'Océanie française. Ces politiques opèrent des différentiations en fonction des populations rencontrées, du contexte dans lequel elles se trouvent, des caractéristiques "raciales " que les officiels français leur attribuent ou encore des rapports de force instaurés localement. Mais nous verrons que l'analyse comporte bien plus de nuances et d'ambiguités que la simple opposition entre des Polynésiens éduqués sous Pomaré, qui se seraient offerts à la domination française, et les Kanak ou Marquisiens pour lesquels l'accès à la citoyenneté relèverait d'emblée de l'impensable. En effet, l'extension de la citoyenneté aux sujets de Pomaré s'accompagne d'un élargissement de la pratique du vote dans des lieux aussi improbables que les Marquises et les Gambier, au nom de la cohérence géographique et administrative des Établissements d'Océanie. En revanche, les Kanak, dès la prise de possession française en I853, sont considérés comme des primitifs dont les pratiques, en particulier l'anthropophagie, les condamnent, et pour lesquels

Sylvie Thénault, "L'indigénat dans l'empire français : Algérie/Cochinchine, une double matrice, Monde(s), $\mathrm{n}^{\circ} \mathrm{I} 2,20 \mathrm{I} 7$, p. $2 \mathrm{I}-40$.

6. Jean-François Baré, "La France dans la longue durée tahitienne", Revue française d'histoire d'Outre-Mer, $\mathrm{n}^{\circ}$ 284-285, I989, p. 63-93. Voir aussi Jean-François Baré, Tahiti, le temps et les pouvoirs. Pour une anthropologie politique du Tahiti post-Européen, Paris, Orstom, I987.

7. Anne-Christine Trémon, "Citoyens indigènes et sujets électeurs. Statut, race et politique dans les Établissements français de l'Océanie (I880-1945) ", Genèses, n ${ }^{\circ}$ 9I, 2013/2, p. 28-48 ; Colin Newbury, "La représentation politique en Polynésie française, I880-1903", Fournal de la Société des Océanistes, $\mathrm{n}^{\circ} 23, \mathrm{I} 967, \mathrm{p}$. II-27. 
s'impose à l'évidence le statut d'indigène non citoyen et, par voie de conséquence, la soumission au régime de l'indigénat. Pourtant, on doit noter que les Kanak n'ont pas le monopole de coutumes que les Européens réprouvent. Les Marquisiens pratiquaient aussi l'anthropophagie et les Tahitiens, adeptes du culte d'Oro, étaient célèbres pour leurs sacrifices humains et le recours à l'infanticide ${ }^{8}$. Il faut aussi rappeler qu'à Tahiti comme en Nouvelle-Calédonie, les indigènes ne sont pas seuls en cause dans le débat qui s'organise à la fin du siècle sur les enjeux de la représentation politique. Les colons, Français citoyens, installés localement, réclament eux aussi le droit d'élire leurs représentants. C'est dans ce contexte compliqué que se joue l'extension des principes démocratiques de la France de la III $^{\mathrm{e}}$ République vers les territoires du Pacifique, contexte que nous voudrions ici revisiter pour mieux comprendre les logiques d'inclusion ou d'exclusion des indigènes dans la citoyenneté et la pratique du vote en situation coloniale. Les territoires de Tahiti et de la Nouvelle-Calédonie sur lesquels nous concentrons notre attention sont les pivots incontournables d'un schéma politique appliqué par la France en Océanie 9.

\section{La France face à ses nouveaux sujets en Océanie : I840-I880}

\section{Les ambigüités du protectorat français à Tahiti}

Rappelons que l'État français entre en Océanie à la fin des années I830, dans le sillage des missionnaires catholiques picpusiens qui cherchent à s'installer à Hawaï, à Tahiti, aux Marquises et aux Gambier, tandis que leurs confrères, les Maristes, font de même à Wallis et Futuna, à Tonga puis en Nouvelle-Calédonie. Les Marquises sont annexées en 1842 , un protectorat est imposé à la reine Pomaré ro à Tahiti la même année, tandis que l'annexion de la Nouvelle-Calédonie, tentée une première fois en I843, est confirmée en I853. L'influence française s'impose à Wallis et Futuna dans les années 1840 et se transforme en protectorat dans les années I880. Elle s'impose dans le même temps aux Gambier et aux îles Australes qui sont annexées formellement par la France en I88I.

8. Le culte d'Oro, dieu de la guerre et du tonnerre, parti de l'île Raiatea dans les années I740, s'imposa rapidement dans les îles avoisinantes, Tahiti, Marquises, Cook, Australes, Tuamotu, diffusé par les membres de la caste des Arioi, spécialistes des rituels, chants et danses dédiés à ce culte singulier. Ce culte, perçu parfois comme un véritable mouvement missionnaire et colonisateur, dominait l'île de Tahiti lorsque les Européens y accostèrent à la fin du $\mathrm{XvIII}^{\mathrm{e}}$ siècle.

9. Pour un développement détaillé voir Isabelle Merle et Adrian Muckle, L'Indigénat, op. cit., p. 7I-I2O.

IO. Aimata (I8I3-I877) est la fille du chef (Ari'i) Pomaré II et demi-sœur de Pomaré III que les missionnaires évangéliques britanniques, présents à Tahiti depuis 1797 intronisent en I823 en grande pompe comme " roi " sur le modèle de Westminster. À la mort précoce de ce dernier en I827, Aimata lui succède en tant que " reine " du Royaume dit de Pomaré recouvrant l'île de Tahiti, Morea et une partie des Tuamotu. 


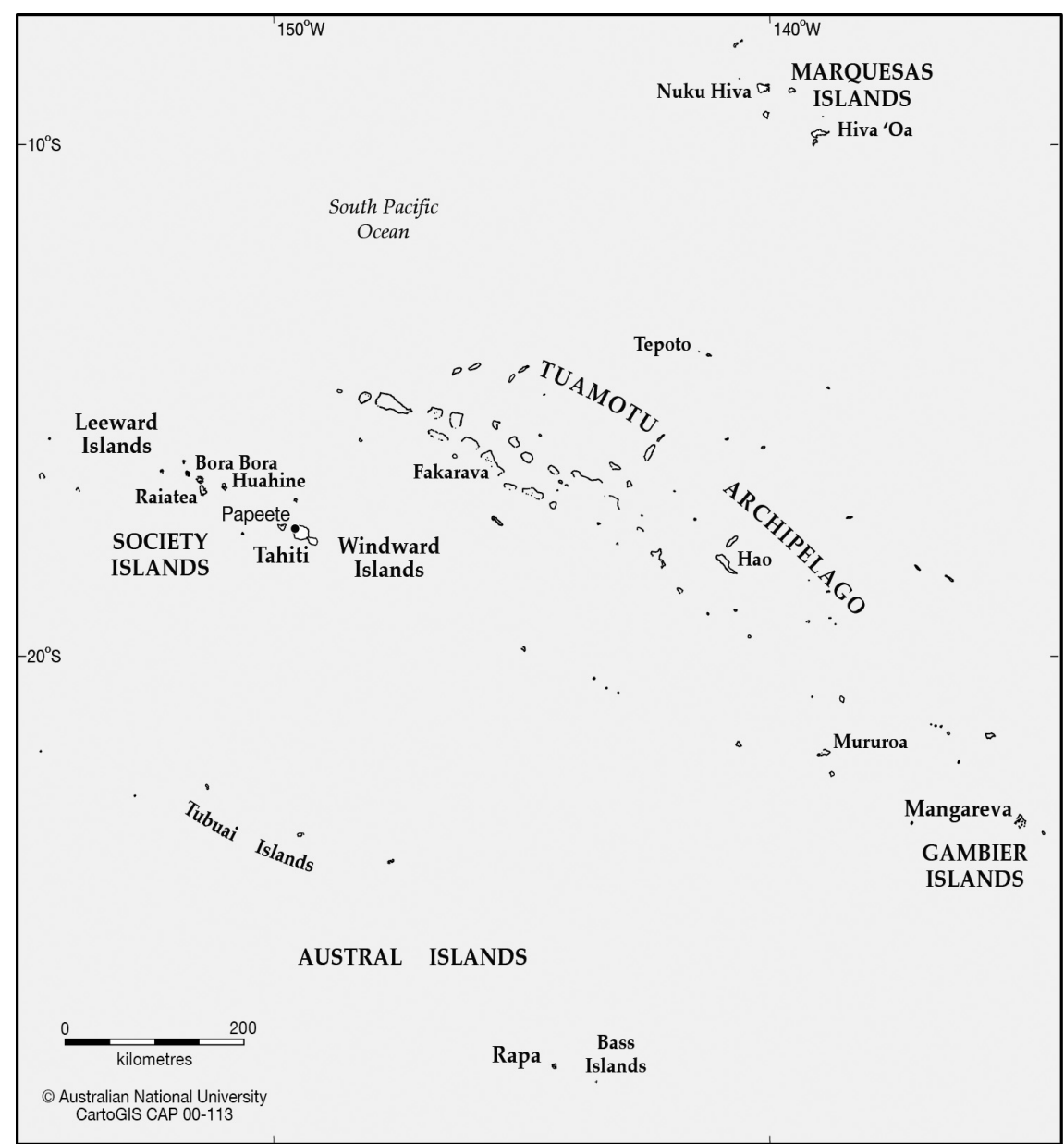

Figure I : Polynésie française.

On note ici la relative unité de lieu et de temps qui caractérise les conquêtes françaises en Océanie. Celles-ci s'opèrent précocement, alors que la France mène dans le même temps la guerre en Algérie entre I840 et I850, et progresse en Asie à partir de I860. Contrairement à ce qu'on pourrait penser, les territoires du Pacifique offrent de sérieuses résistances, aux Marquises d'abord, dès 1842, à Tahiti en I844-I845 et en Nouvelle-Calédonie, dès I855 et au cours de deux décennies suivantes. Cette résistance armée encourage la Marine française et les gouverneurs, qui sont alors issus de ses rangs, à exiger pour l'ensemble de ces territoires une organisation administrative et judiciaire au cœur de laquelle se trouve la figure d'un gouverneur doté de pouvoirs spéciaux et extra-judiciaires. Cette organisation fondée sur l'ordonnance du 28 avril I843, conçue pour les Marquises, étendue en 
I845 à Tahiti puis en I855 à la Nouvelle-Calédonie, s'inspire directement de l'expérience algérienne et plus précisément de l'arrêté du $\mathrm{I}^{\text {er }}$ septembre $\mathrm{I} 834$ dont le principal objectif était de consolider les pouvoirs d'un gouverneur militaire tout puissant. Pour autant, la France rencontre dans ces territoires océaniens des situations locales très différentes, plus ou moins marquées par les intrusions européennes antérieures et en particulier la présence britannique.

C'est à Tahiti qu'elle est obligée de composer le plus avec des chefferies christianisées par des missionnaires évangéliques, protestants et britanniques, installés depuis la fin du $\mathrm{xvIII}^{\mathrm{e}}$ siècle, et dont l'influence ne s'est pas limitée à l'expansion du message du Christ, mais a modifié en profondeur les univers politiques locaux et les organisations sociales. L'alliance entre la chefferie Pomaré et les premiers missionnaires débarqués sur l'île au Vent (Tahiti) en I797, a conduit à la centralisation du pouvoir aux mains de cette chefferie, au détriment des équilibres anciens fondés sur les logiques segmentaires et hautement compétitives entre groupes ou lignages de force équivalente. La soumission progressive de l'ensemble des chefferies à la suprématie des Pomaré fonde ce que les Britanniques vont appeler "le Royaume de Pomaré " ${ }^{\text {II. Les }}$ évolutions ne s'arrêtent pas là, puisqu'à partir de I8I9 sont adaptées localement un certain nombre d'institutions inspirées par le droit britannique et les valeurs chrétiennes : le code de lois pénales et civiles dit code Pomaré, le corps des "grands juges " (To'ohitu) et l'assemblée tahitienne créée en I824 sur la base de chefs et juges élus par ceux que les missionnaires voient comme les propriétaires des terres, les Ra'atira. Lorsque les Français s'imposent en I842, ils font face à l'hostilité d'une partie des élites polynésiennes, la reine Pomaré en tout premier lieu, profondément attachée aux normes et valeurs d'un monde polynésien anglophone et imprégnée par la religion protestante.

La France mène la guerre aux Marquises en I842-I844 et à Tahiti en I844-I845. Dans les deux cas, il s'agit d'imposer par la force la soumission des chefs, mais le commandant Bruat choisit à Tahiti de composer avec une société tahitienne qu'il admire ${ }^{\mathrm{I} 2}$. En échange de la promesse

II. Jean-François Baré, Le Malentendu pacifique. Des premières rencontres entre Polynésiens et Anglais et ce qui s'ensuivit avec les Français jusqu'à nos jours, Paris, Éditions des archives contemporaines, 1985; Colin Newbury, Tahiti Nui, Change and Survival in French Polynesia, I767-I945, Honolulu, The University of Hawaii Press, 1980.

I2. Comme en témoigne le mémoire qu'il rédige pour son successeur, le capitaine de vaisseau Lavaud en 1849: Mémoire de Bruat, Archives nationales d'Outre-Mer (ANOM), Fonds ministériel, Séries géographiques, OCEA/I/47. Voir Colin Newbury, "Le commandant Bruat et les chefferies tahitiennes, fournal de la Société des Océanistes, I955, II, pp. 5-I3. Le Commandant Armand Joseph Bruat (I796-I855) est envoyé en Océanie en I843, nommé premier gouverneur des îles Marquises qui ont été annexées par la France l'année précédente et Commissaire auprès de șa majesté, la reine Pomaré, à Tahiti où il est chargé d'instaurer le protectorat français. Étant parvenu à imposer par la force la souveraineté française dans ces deux territoires, il quitte l'Océanie en I849 pour devenir gouverneur des Antilles où il entend faire respecter l'ordre public colonial dans le contexte de l'abolition de l'esclavage. 
qui est faite de protéger les terres et titres fonciers, les chefferies sont invitées à rallier le protectorat français dont Bruat est en train d'inventer localement une forme originale, faute de pouvoir s'appuyer sur des expériences antérieures, si ce n'est l'exemple britannique des îles Ioniennes en I8I 5 et les traités de Casamance signés par la France en I838-I839 ${ }^{\text {I3 }}$. La formule du protectorat colonial est loin d'être stabilisée, et Bruat bricole son propre modèle tahitien en décidant de s'appuyer sur l'organisation héritée : la Reine, les chefs, les juges et l'assemblée tahitienne. Il promet "de prendre en considération les droits et prétentions et les influences politiques de chaque famille importante dans le but de favoriser les partisans pro-français sans contrevenir aux coutumes " ${ }^{14}$ et s'appuie sur l'assemblée tahitienne pour gratifier les chefs, désormais rémunérés par l'État. Mais la convention rédigée par son successeur en 1847 entend bien réformer les institutions locales et, en tout premier lieu, le statut des chefs. Les anciens tributs offerts par les sujets sont supprimés ainsi que les hiérarchies de rang. Les chefs seront désormais des agents publics égaux chargés d'appliquer les arrêtés et décisions du gouverneur et de maintenir l'ordre public local grâce à leur police dont les membres, les mutoï, seront nommés et rémunérés eux aussi par l'État, comme les juges. L'assemblée tahitienne est maintenue mais, dès I848, il est exigé qu'elle adopte les modes de fonctionnement et les procédures du Parlement français. Le ministre de la Marine espère ouvertement que cette convention de I847 sera un moyen "de galliciser les Tahitiens " tout en maintenant les apparences du respect de l'autonomie interne, des institutions et coutumes :

Au moyen de l'influence active que vous serez capable d'exercer sur la Reine et les Chefs, par la confiance que vous leur inspirerez, vous les habituerez rapidement à vous voir participer de plus en plus à l'administration interne. Ainsi, le Protectorat deviendra une institution plus apparente que réelle sous laquelle la loi souveraine sera progressivement établie ${ }^{15}$.

La défiance à l'égard de ces institutions tahitiennes ne tarde pas à s'installer, comme en témoigne une note attribuée au gouverneur du Bouzet ${ }^{16}$, en charge des Établissements de l’Océanie entre I854 et I858 :

13. Isabelle Surun, "Une souveraineté à l'encre sympathique ? Souveraineté autochtone et appropriations territoriales dans les traités franco-africains au XIx ${ }^{\mathrm{e}}$ siècle", Annales HSS, 2014-2, p. 313-348.

I4. Colin Newbury, Tahiti Nui, op. cit., p. I25, notre traduction.

I5. Ibid., p. I27.

I6. Joseph, Fidèle, Eugène, Marquis du Bouzet (I805-1867), officier de marine, est mis au service de la station navale du Pacifique à partir de I84I pour soutenir l'installation des missionnaires catholiques français en Nouvelle-Zélande, à Wallis et Futuna, aux Marquises, à Tahiți, en Nouvelle-Calédonie, à Tonga et Samoa. Entre I854 et I858, il est gouverneur des Établissements de l'Océanie française qui recouvrent les territoires français de Polynésie et la Nouvelle-Calédonie. En I860, la Nouvelle-Calédonie se voit doter d'une administration et d'un gouverneur autonome. 
Par la force des choses et contrairement au traité du Protectorat notre action sur le gouvernement intérieur de ces îles s'est constamment étendue ; l'indolence des Indiens, leur manque absolu de principes et l'incapacité de la Reine font que, si elle exerçait seule ce pouvoir, la machine ne tarderait pas à ne plus pouvoir fonctionner [...]. La Reine malgré toutes les démonstrations extérieures a toujours conservé un fond de rancune contre nous. Elle est portée à subir l'influence des mauvais conseils et ne rend justice à notre politique désintéressée que quand elle a besoin de nous. La sienne est de chercher à rétablir le plus qu'elle pourrait les anciens abus. Je ne crois pas que les Indiens l'aiment au fond car elle ne s'occupe d'eux que pour les dépouiller. ${ }^{17}$

Du Bouzet dénonce "les Indiens qui n'ont d'ailleurs aucun ordre d'idées et ne sont jamais pressés " et les dysfonctionnements d'une assemblée tahitienne dont il organise les sessions avec de plus en plus de retard sans même que les chefs, cheffesses ou juges tahitiens qui la composent s'en aperçoivent.

Les Indiens aiment toujours à faire de l'opposition ; elle n'est pas raisonnée, mais en général, toutes les mesures utiles qu'on propose passent sans trop de difficulté. Il y aurait de l'inconvénient à faire durer l'assemblée plus de quinze jours car cela dérange beaucoup les Indigènes et la prolongation de leur séjour à Papeete leur est funeste ; ils se livrent avec excès à la boisson et font des sottises. On est quelquefois obligé d'en expulser de l'assemblée pour cause d'ivresse ${ }^{18}$.

On mesure ici toute l'ambigüité du modèle de protectorat français qui sert de vitrine pour manipuler les instances représentatives tahitiennes et imposer progressivement les normes et pratiques du droit et de l'administration française. Ces dernières sont étendues dans les années I850 avec la mise en place d'impôts et de corvées de route. Elles sont encore renforcées avec l'arrivée au poste de Gouverneur, en I864, d'Émile de la Roncière, en provenance d'Algérie où il fut inspecteur général. Fervent défenseur de l'assimilation juridique, Roncière étend la compétence des tribunaux français à tous les habitants de Tahiti et réduit d'autant la compétence des tribunaux dits indigènes, désormais limitée aux seuls conflits fonciers entre Tahitiens. En I868, il soumet l'ensemble de la population tahitienne aux règles du Code Civil. Les Tahitiens, considérés comme des sujets du droit civil français, ne se verront pas appliquer ce qu'on appellera le statut personnel réservé aux peuples colonisés dont la France prétend reconnaître les mœurs et coutumes particulières.

Roncière voudrait défendre l'autonomie d'un royaume tahitien sur la base d'un conseil mixte composé de Tahitiens et d'Européens, doté d'un budget autonome et d'un pouvoir législatif, avec, à ses côtés, un

I7. Note non signée adressée de la Nouvelle-Calédonie par M. le Gouverneur des Établissements français de l'Océanie, du Bouzet, parvenue à Tahiti le 3 février I860.

ANOM, Fonds ministériels, Séries géographiques, OCEA/35/107.

I8. Ibid. 
Conseil général exclusivement européen et seulement consultatif. Mais, sous la pression des colons locaux, son projet est refusé. Il est immédiatement rappelé à Paris et laisse surtout, derrière lui, les acquis d'une politique de francisation qui ne cesse de s'affirmer, du moins dans les textes. Car, sur le terrain, on s'en doute, le processus est beaucoup plus complexe et aléatoire. Les gouverneurs ne restent pas longtemps et maitrisent mal les subtilités du pays. Le pouvoir, en pratique, est le fruit d'un compromis local entre chefferies, colons, missionnaires et fonctionnaires subalternes résidant suffisamment de temps à Tahiti pour pratiquer la langue Mao'hi. La dynastie Pomaré domine de facto un réseau de chefferies qui lui ont fait allégeance à Tahiti (île au Vent) et jusqu'à Tubuaï et l'ouest des Tuamotu. Elle reste étroitement liée aux grandes familles de Raïatéa, Bora Bora, Tupai et Huahine (îles Sous-le-Vent) mais celles-ci, fidèles aux missionnaires britanniques, ont obtenu en I847, grâce aux rivalités franco-britanniques, un statut d'indépendance qu'elles ne perdront qu'en I887. Les unes et les autres partagent un même socle socio-culturel et linguistique $M a o^{\prime} h i$ dont la vitalité persiste dans les districts, à l'ombre des paroisses protestantes sous l'autorité des diacres et des chefs, et à l'assemblée lorsqu'il s'agit de choisir les représentants au sein des lignages prestigieux. Les sujets du roi Pomaré se familiarisent néanmoins progressivement avec les règles administratives, au contact des fonctionnaires et de la langue française, par exemple lors de la lecture des arrêtés et règlements, pour certains non traduits en tahitien, et les chefs s'habituent à la rente financière que leur procure le nouvel ordre administratif. Mais le nouveau pouvoir ne peut ignorer le contexte polynésien très singulier qui l'entoure, l'héritage politique laissé par les évangélistes protestants ou encore l'ébauche de pratiques parlementaires autochtones.

\section{L'entrée de la France en Nouvelle-Calédonie et l'usage du rap- port de force}

Toute autre est l'expérience française en Nouvelle-Calédonie, qui s'amorce en I853 avec la prise de possession déclarée sur le lieu-dit de Balade, au nord-est de l'île. Ici, nul besoin de composer avec un ordre politique déjà influencé par l'Europe. La chefferie Puma, dominant la région de Balade, n'ignore pourtant pas les Blancs. Elle avait vu débarquer Cook en 1774, puis d'Entrecasteaux en I893, et a accueilli en I843, avec ses voisins de Pouébo, les premiers pères maristes, violemment chassés en I847. Elle sait traiter, comme les autres chefferies du bord de mer sur la côte est, avec les négociants anglophones venus chercher le santal pour le revendre sur le marché chinois ${ }^{19}$.

I9. Isabelle Merle, Expériences coloniales. La Nouvelle-Calédonie. I853-1920, Paris, Belin,I995; Michel Naepels, Conjurer la guerre. Violences et pouvoir à Houailou (Nouvelle- 


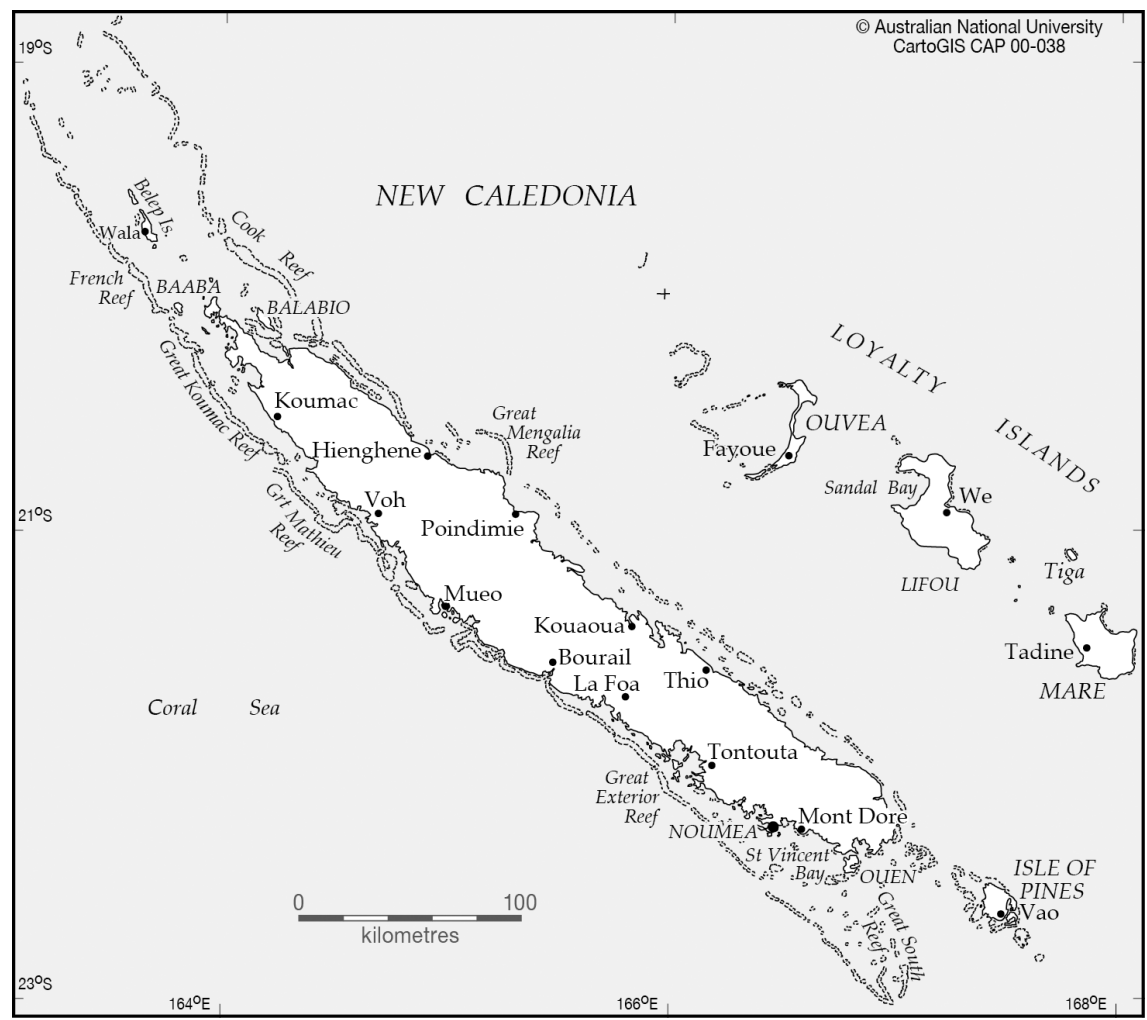

Figure 2 : Nouvelle Calédonie.

Mais lorsque l'ordre est donné, le 23 avril I853, au contre-amiral Febvrier des Pointes ${ }^{20}$ et à l'officier Tardy de Montravel ${ }^{21}$, de prendre solennellement possession de la Nouvelle-Calédonie en deux points, il n'est prévu de négocier ni traité, ni cession de territoire, pas plus qu'il n'est prévu d'obtenir la signature attestant le consentement des chefs sur le document actant la prise de possession, exception faite du chef Vendegou de l'île des Pins. Tardy de Montravel tient à confirmer les actes de prise de possession par des reconnaissances de souveraineté obtenues auprès des principaux chefs de la côte est. En échange de la protection que leur confère leur nouvelle nationalité, ces derniers doi-

Calédonie), Paris, Éditions de l'EHESS, 2013 ; Isabelle Merle et Adrian Muckle, L'indigénat, op. cit.

20. Le contre-amiral Febvrier des Pointes (I796-I855) est nommé en I85I Commandant naval d'Océanie et des Côtes occidentales d'Amérique.

2I. Louis Marie Tardy de Montravel (I8II-I864), officier de marine, était chef de la division navale de la Réunion et des Indes lorsqu'il fut appelé pour confirmer l'acte de prise de possession déjà signé par Febvrier des Pointes à l'île des Pins, le I8 janvier I854. 
vent promettre de protéger les sujets français et les étrangers ${ }^{22}$. Les 7 et I5 février I854, les chefs de Balade et Pouébo affirment ne plus appliquer désormais à leurs sujets, "devenus sujets français, les lois et coutumes du pays " ${ }^{23}$. Ce renoncement est justifié par la volonté de faire "participer [la tribu] aux droits et avantages de tous citoyens français" et "de ne pas la laisser dépourvue de toute espèce de code " 24. Mais "les droits et devoirs de tous citoyens français" sont immédiatement oubliés alors qu'une liste d'interdits est dressée : le meurtre suivi d'anthropophagie, le meurtre par vengeance, le meurtre des vieillards et infirmes, la guerre, le vol, l'incendie, les coups et insultes graves, l'adultère, le viol et tout attentat aux mœurs, la désobéissance aux chefs, le faux témoignage, les danses nocturnes.

Les interactions entre les autorités coloniales françaises et les chefs kanaks sont ainsi placées d'emblée sous le régime de la force et de la répression. En I855, du Bouzet étend à la Nouvelle-Calédonie l'ordonnance déjà appliquée aux Marquises et à Tahiti en ce qui concerne les pouvoirs "spéciaux » du gouverneur. Et sa politique envers les Kanak se durcit encore, alors qu'éclatent en divers point de la Grande terre des affrontements violents. Le cycle implacable de la guerre et de la répression s'enclenche pour ouvrir la voie à la stratégie de refoulement des populations locales qui se conclura à la fin du siècle par l'organisation de véritables réserves indigènes.

\section{Citoyens ou sujets : enjeux et limites des modes de gouver- nance et de leurs variations}

\section{L'influence d'un modèle colonial autoritaire calédonien}

La Nouvelle-Calédonie fut séparée des Établissements français d'Océanie en I860 lorsqu'il fut décidé de lui octroyer un poste de gouverneur à part entière. L'attraction que suscitait, auprès de migrants, la promesse de terres riches et disponibles sous un climat tempéré ainsi que le projet d'y installer rapidement un établissement pénitentiaire pour doubler celui de la Guyane française, exigea l'autonomie de ce territoire et l'organisation d'un régime administratif propre. De fait, le bagne calédonien fut ouvert en I864 et il parut essentiel, au cours des dix années suivantes, de réformer les institutions pour faire face aux nouveaux enjeux locaux qu'exigeaient la surveillance d'une population kanak insoumise, et tout autant sinon davantage, celle d'une population pénale potentiellement dangereuse.

22. Acte de reconnaissance de souveraineté, Bulletin officiel de la Nouvelle Calédonie $(B O N C), 23$ janvier 1854, p. 9.

23. BONC, 7 février I854, p. Io ; BONC, I5 février I854, p. I6-I7.

24. Ibid. 
Parmi les réformes les plus importantes figure la promulgation, le I2 décembre 1874, d'un texte fondamental qui règle en I67 articles le fonctionnement et les attributions des principaux services de l'administration locale. 95 articles sont consacrés au seul gouverneur qui dispose d'un pouvoir sans contrepartie, si ce n'est le contrôle de l'autorité du ministre et la consultation éventuelle du conseil privé dans certains domaines ${ }^{25}$. Ce texte vise à remplacer l'ordonnance de I 843 qui s'était avérée notoirement insuffisante.

Ce texte fait l'admiration des autorités coloniales de Tahiti lorsqu'en I876 elles se plaignent des lacunes du protectorat : "Toutes les colonies, à ce jour, même la Nouvelle-Calédonie, qui n'était autrefois qu'une dépendance de Tahiti, ont l'avantage de posséder une organisation régulière " 26 . Une commission est instituée à cet effet qui, dans ses conclusions, distingue clairement les politiques qui doivent être menées dans les deux colonies mais témoigne aussi d'une réelle ambivalence à l'égard des Polynésiens. Il s'agit d'insister à la fois sur "la différence profonde " existant "entre l'esprit, le but, l'intention du législateur tahitien et néo-calédonien " ${ }^{27}$, en s'écartant d'un modèle de gouvernement très autoritaire, jugé nécessaire pour la Nouvelle-Calédonie mais excessif pour Tahiti, et de se prémunir contre les contestations et hostilités "anti-françaises " qui n'ont jamais disparu à Tahiti et qui peuvent advenir dans les périphéries du Royaume comme les Gambier, qu'elles proviennent des Polynésiens eux-mêmes ou des étrangers résidant dans les îles. Le rapport de la commission exige "quelque chose de moderne " pour les Établissements d'Océanie mais veut s'inspirer de l'organisation du gouvernement en Nouvelle-Calédonie. Il demande en particulier le rétablissement à Tahiti du titre et de la fonction de gouverneur et la consolidation de l'ensemble de ses pouvoirs. Il insiste cependant sur ce qui oppose les situations dans lesquelles se trouvent respectivement les deux colonies : le fonctionnement du régime du protectorat et la naissance "d'intérêts locaux " à Tahiti ; " un pays affecté à la transportation et à la déportation" en Nouvelle-Calédonie ${ }^{28}$.

Cette reconnaissance des intérêts locaux, plus précisément ceux des colons, à Tahiti, se traduit par la prudente ouverture du Conseil d'administration de la colonie à quelques notables européens, mais il n'est pas encore question de membres élus, pas plus qu'il n'est question d'y introduire des personnalités indigènes à l'égard desquelles le président de la commission éprouve une grande méfiance :

25. Arrêté du I ${ }^{\mathrm{er}}$ mars I875. Promulgation du décret organique du I 2 décembre $\mathrm{I} 874$ concernant le gouvernement de la Nouvelle-Calédonie, fONC, I875, p. I 2.

26. Rapport du président de la commission chargée d'étudier les réformes administratives, Papeete, le 4 décembre I876, ANOM, Fonds ministériels, Séries géographiques, OCEA/35/107.

27. Ibid.

28. Ibid., Projet d'organisation coloniale au ministre, I5 octobre I878. 
La Royauté tahitienne, écrit-il, n'est que le masque cherchant à cacher toutes les figures qui peuvent être hostiles à la France [...] des gens qui nous sont étrangers à tout point de vue [...]. Il faut ici rappeler les raisons qui ont fait demander le protectorat, [...] Ils se déclarent incapables et demandent à se mettre en tutelle. Ils sont en état de minorité ! et on voudrait aujourd'hui [...] admettre les protégés à gouverner les protecteurs, les mineurs à gouverner les tuteurs ${ }^{29}$.

La vision d'un pouvoir tahitien irresponsable et rendu totalement impuissant (tous les actes de la Royauté devant recevoir l'aval du gouverneur) justifie la proposition par la commission d'un projet de décret proche du modèle calédonien, prévoyant notamment le contrôle rigoureux d'une presse écrite qui risquerait de "frapper l'imagination des indigènes ", "dans un esprit anti-français " $3^{\circ}$, et le maintien des pouvoirs spéciaux au gouverneur, en cas de menace pour la sécurité publique. A la différence de la Nouvelle-Calédonie, cependant, le gouverneur à Tahiti doit dans ce cas consulter son Conseil d'administration et se conformer à son avis. Il doit aussi tenir compte " du respect de la liberté individuelle " en limitant la détention extra-judiciaire à dix jours, l'assignation à résidence à un an et l'exil des sujets du Royaume à trois ans ${ }^{3 \mathrm{I}}$.

Pourtant, le projet de décret soumis au ministre par le Conseil d'administration de Tahiti en 1878 reste lettre morte, et la nécessité d'une réforme de l'organisation administrative du territoire est réaffirmée par le Commissaire Chessé lorsqu'il décide d'accélérer le processus d'annexion française en négociant directement avec le roi Pomaré et les principaux chefs de l'île, le 29 juin I880, l'abandon de la souveraineté pleine et entière du royaume à la France ${ }^{32}$.

Encouragé par son autorité de tutelle, il fait miroiter au roi des projets de réformes dont il vante le caractère libéral : la création d'un conseil colonial mixte, moitié français, moitié indigène, et d'un conseil tout indigène, à laquelle s'ajoutent la promesse d'une augmentation substantielle du traitement des chefs, celle de rentes viagères dont bénéficieraient tous les membres de la famille royale ainsi que l'effacement des dettes laissées par la Reine Mère Pomaré IV 33.

Il y a, dans la pression qu'exerce Chessé sur le roi Pomaré V une part indéniable de marchandage. Il tient à flatter, chez le Roi, son souci du

29. Projet d'organisation coloniale, $\mathrm{I}^{\mathrm{er}}$ mai $\mathrm{i} 878$, introduction du président de la commission. ANOM, Fonds ministériels, Séries Géographiques, OCEA/35/107.

30. Ibid.

31. Ibid. Discussion autour de l'article 69 sur les pouvoirs extraordinaires du gouverneur.

32. Isidore Henri Chessé (I839-19I2), militaire de carrière est gouverneur des Établissements français d'Océanie, brièvement, en I880-I88I, le temps d'annexer Tahiti à la France et d'obtenir ainsi la légion d'honneur. Il poursuit sa carrière de gouverneur en Guyane en I883-I884.

33. Lettre du commandant commissaire Chessé au ministre des Colonies, le 5 juillet I880. ANOM, Fonds ministériels, Séries géographiques, OCEA/I39. 
prestige et de l'étiquette, et à promettre de généreux subsides. Mais il veut aussi rassurer les élites polynésiennes par la promesse de réformes politiques garantissant l'exercice de la représentativité, le contrôle exercé sur les terres par les tribunaux indigènes et le développement des écoles. Le ralliement, le 29 juin I 880 , du chef de Faaa, pasteur marié à la fille du colon anglophone Salmon, l'homme le plus éloquent de Tahiti, où ils le sont tous selon Chessé, est décisif et encourage l'ensemble des chefs à signer la déclaration qui " remet et pour toujours entre les mains de la France, le gouvernement et l'administration de nos États comme aussi tous nos droits et pouvoirs sur les iles de la Société et Dépendances" 34 .

Le vote de la loi par le Parlement français, le 27 décembre I 880 , déclare l'île de Tahiti et ses dépendances, colonie française. En contrepartie, "la nationalité française est acquise de plein droit à tous les anciens sujets du Roi de Tahiti " 35. Le Roi se verra accorder une rente annuelle et sera libéré de toutes les dettes laissées à la mort de sa mère.

\section{La question de la représentativité en colonie : pour "nous " ou pour " eux "}

Pour comprendre les conditions dans lesquelles la loi de I880 est promulguée, il faut revenir sur la montée des revendications appelant à la représentation politique des colons, lesquelles se font jour non seulement à Tahiti, mais aussi en Nouvelle-Calédonie, au cours de la décennie qui précède. Les Français citoyens installés dans ces deux territoires, parce qu'ils payent des impôts, s'estiment de plus en plus légitimes à élire des représentants pour la gestion des affaires de leur colonie et à obtenir un pouvoir délibératif sur les dépenses du budget colonial ${ }^{36}$.

Une première pétition est signée par des colons et commerçants de Nouméa, dès I873, pour réclamer un Conseil colonial capable de les représenter et un Conseil municipal élu dans la principale ville du pays. En I876, une deuxième pétition exige la création d'un Conseil général élu sur le modèle ceux qui fonctionnent à la Réunion, à la Martinique ou en Guadeloupe. Il va de soi, pour les pétitionnaires de NouvelleCalédonie, que leurs revendications ne concernent en rien la population indigène kanak qui n'est aucunement évoquée. À Tahiti, en revanche, une note rédigée par le président du Comité central de

34. Déclaration de prise de possession du Royaume de Pomaré par la France, 29 juin I880. Ibid.

35. Ibid.

36. La population de Français citoyens, à Tahiti, s'élève à environ 800 personnes dans les années I880, installés en très grande majorité à Papeete. Voir PierreYves Toullelan, Tahiti colonial, I860-I9I4, Paris, Publications de la Sorbonne, 1984. En Nouvelle-Calédonie, le recensement de I887 indique 906I personnes d'origine libre et Io 726 personnes d'origine pénale. Voir Isabelle Merle, Expériences coloniales. La NouvelleCalédonie, 1853-1920, Paris, Belin, 1995. 
l'agriculture et du commerce à Tahiti, adressée le I8 janvier I 880 au sénateur Victor Schoelcher, exige des droits égaux à ceux que sont supposés avoir les Tahitiens. Faisant référence aux procédures électives appliquées aux Tahitiens sous le protectorat, elle précise : "Les Tahitiens nomment à l'élection leurs chefs, leurs conseillers municipaux, leurs maîtres d'école, leurs ministres protestants, leurs députés, leurs juges de la Cour suprême. Les Français [de Tahiti] ne jouissent d'aucune de ces prérogatives et d'aucun de ces droits " 37. La même année, un article signé par Albert Rabou, intitulé "Les réformes coloniales " et publié dans un journal parisien, affirme au contraire que l'administration de Tahiti a annulé la représentation des Tahitiens en refusant de convoquer l'assemblée tahitienne. "Le commandant français est maître absolu du service indigène et du budget indigène que des officiers, relevant de lui seul, administrent sans aucun contrôle. $C e$ sont les fameux bureaux arabes de l'Algérie transportés en Océanie " ${ }^{8}$. Les colons, quant à eux, ne jouissent d'aucun de leurs droits de citoyens français. Ils sont, par rapport au commandant, "dans la situation des paysans vis-à-vis leur seigneur avant 1789 " 39. Une pétition est également signée le 29 mars 1880 à Tahiti pour soutenir la demande d'un conseil administratif élu chargé de voter le budget.

L'idée d'adjoindre des représentants de la société civile au Conseil d'administration ou au Conseil privé est apparue précocement dans les deux colonies, avec la nomination en Nouvelle-Calédonie de citoyens français dès I855 (à l'exclusion donc des sujets indigènes) et de représentants indigènes et européens à Tahiti, dès I86I, puis à nouveau en I870. Mais dans les deux cas, la proposition d'ouverture que font les gouverneurs reste prudemment limitée à des membres nommés et n'envisage qu'avec beaucoup de circonspection, la formation d'un corps local souverain d'électeurs et d'élus. Les gouverneurs se méfient des colons qu'ils jugent trop peu nombreux et peu instruits 40. En Nouvelle-Calédonie, le gouverneur Pritzbüer n'hésite pas à qualifier de tarée "ce qu'on appelle la population libre" 4 r et, à Tahiti, on craint surtout les stratégies d'hommes entièrement dévoués à leurs propres intérêts agitant au surplus les passions religieuses conflictuelles entre

37. Note du président du Comité central d'agriculture et du commerce de Tahiti à M. le sénateur Schœlcher sur la situation actuelle de Tahiti. Neuilly I8 janvier I880, ANOM, Fonds ministériels, Séries géographiques, OCEA/Ioo.

38. Copie d'un article trouvé dans les archives d'Albert Rabou intitulé «Les réformes coloniales ", 22 février I 880 , publié dans le 23 février dans un journal parisien dont on ne peut voir le nom. Ibid.

39. Ibid.

40. "L'application directe du suffrage universel à l'élection des Conseillers peut amener au Conseil des habitants d'une instruction négative et d'une valeur nulle ", Lettre du I5 octobre I878 concernant le projet d'organisation coloniale de Tahiti adressée au Ministre par le Commissaire résident. ANOM, Fonds ministériels, Séries géographiques, OCEA/35/107.

4I. Citée dans Pierre Gasher, La belle au bois dormant. Regard sur l'administration coloniale en Nouvelle-Calédonie, 1874-I894, Publications de la SHNC, n ${ }^{8}$, I975, p. I07. 
protestants (à majorité indigène) et catholiques (à majorité européenne). Les relations ultérieures houleuses entre représentants de l'État et élus des Conseils généraux (Conseiller colonial à Tahiti, jusqu'en I884) confirmeront cet état de méfiance persistant allant jusqu'à la menace de dissolution des instances et parfois sa mise en œuvre $4^{2}$.

Quoiqu'il en soit, c'est dans ce contexte de revendications démocratiques que se situe l'une des divergences profondes de destin entre la Nouvelle-Calédonie et les Établissement français d'Océanie. Pour les colons calédoniens, il n'y a aucun doute sur les limites du corps souverain qu'ils réclament. Celui-ci ne pourra inclure que les citoyens français et exclure les Kanak. Pourtant, il faut noter que, tout aussi saugrenue que l'idée d'un vote kanak puisse apparaître à un colon local des années I880, celle-ci fait intrusion dans les débats du Conseil général à Nouméa lorsque le directeur de l'intérieur, nouvellement arrivé de Tahiti en I886, se permet d'évoquer la citoyenneté accordée aux sujets de Pomaré. Alors que l'on cherche à imposer aux seuls Kanak le paiement de l'impôt de capitation, un conseiller rétorque que, "d'après la Constitution, tout homme qui paie des impôts a le droit de voter " 43. À la grande surprise de membres du Conseil général de la Nouvelle-Calédonie, le directeur de l'intérieur prête une oreille attentive à cette idée du " droit de vote aux indigènes » lancée sur le mode de la plaisanterie. Un journaliste local qui relate le débat met en garde ce dernier "récemment arrivé de Tahiti, où les indigènes sont parvenus, dit-on à un certain degré de civilisation [...] contre les tendances d'assimilation qui pourraient avoir des conséquences fâcheuses pour le pays qu'il administre aujourd'hui. [...] Lorsque M. le Directeur de l'Intérieur aura parcouru la colonie, qu'il aura visité les tribus [...] alors, il changera certainement d'opinion; il verra l'abîme qui existe aujourd'hui entre l'indigène et le colon, il comprendra combien il serait [dangereux] d'accorder la qualité d'électeur à des hommes qui sont encore nos ennemis " 44 .

L'abîme va se creuser en Nouvelle-Calédonie dans les années qui suivront et il faudra attendre 1932 pour que la possibilité d'accès à la citoyenneté française, accordée en Algérie par le Sénatus-consulte de I865 - via une demande de naturalisation exigeant l'abandon du statut personnel et la conformité au mode de vie français - soit envisagée dans cette colonie du Pacifique ${ }^{45}$.

42. Sur ce point, voir Isabelle Merle, «De la question de la représentativité d'un "peuple citoyen" en Nouvelle-Calédonie. Retour sur l'histoire ", in Carine David (dir.), I5 ans de lois de pays en Nouvelle-Calédonie. Sur le chemin de la maturité, Presses universitaires d'Aix-Marseille, 20I6, p. I9-39.

43. Procès verbaux du Conseil général de Nouvelle-Calédonie, I5 septembre I892, p. 362-363.

44. "Le droit de vote aux indigènes ", L'Indépendant, 6 novembre I 886.

45. Décret du 5 septembre I932, Fournal officiel de la Nouvelle-Calédonie, 5 novembre I932. Le Sénatus-consulte du I4 juillet I865 déclare l'indigène français par nationalité 
Cet abîme, en revanche, n'a jamais existé à Tahiti. Car, dès I880, la discussion sur la question de la représentativité des colons ne peut s'engager qu'au regard d'un héritage déjà marqué par la fiction parlementaire qui s'exerçait au sein de la population tahitienne sous le protectorat et depuis I824. Pour les colons de Tahiti comme pour les autorités locales, il est impossible d'ignorer l'indigène et d'imaginer l'exclure du futur corps électoral d'un conseil général. Le débat est ailleurs et se concentre sur les limites géographiques à donner à ce corps électoral en y incluant l'ensemble des habitants des Établissements français d'Océanie ou seulement les habitants des territoires couverts par l'ancien royaume de Pomaré. Il se concentre aussi sur les conditions de la représentativité politique et les possibilités de sa manipulation, l'enjeu étant d'afficher le principe de l'égalité citoyenne entre Polynésiens et Européens tout en assurant, en pratique, l'influence prédominante des citoyens d'origine française ${ }^{46}$. Le principe du vote, pour ce faire, sera élargi jusqu'aux îles les plus reculées des Établissements français d'Océanie où celui-ci n'a pas de sens.

\section{De la mise à l'épreuve de l'exercice démocratique en situation coloniale : les Établissements français d'Océanie}

«Antérieurement au décret du I4 janvier I860 " peut-on lire dans le rapport de la commission chargée d'établir la réforme administrative à Tahiti en I876, "les îles Marquises, Tahiti et la Nouvelle-Calédonie formaient un seul groupe connu sous la dénomination d'Établissements de l'Océanie et États du Protectorat " 47. La Nouvelle-Calédonie est séparée de ce groupe en 1860 tandis que sont précisées les frontières du Royaume de Pomaré - île au Vent (Tahiti), les Tuamotou, les îles Tubaï et Raivaivae (îles australes) - ensemble administré avec les Marquises sous la houlette d'un même gouverneur. La loi du 30 décembre I880 transforme l'île de Tahiti et les archipels qui en dépendent en colonie et affirme que « la nationalité française est acquise de plein droit à tous les anciens sujets du Roi de Taïti " 48. On note le flou dans lequel est maintenue la qualification géographique des territoires sur lesquels la loi est supposée s'appliquer. Le gouverneur Chessé a joué un rôle majeur dans la transformation du protectorat en colonie mais il étend

mais l'exclut du corps des citoyens au prétexte du maintien de son statut personnel supposé incompatible avec le Code civil. Cependant, il organise une passerelle en proposant aux Algériens l'accès à la citoyenneté par une demande individuelle de "naturalisation" (terme emprunté au droit des étrangers) sur la base d'une enquête de mœurs et sous réserve de se soumettre aux règles du code civil.

46. Vaki Gleizal et Jean-Marc Régnault, "Tahiti et ses îles (I880-I9I4). Une microsociété coloniale aux antipodes de la métropole ", Outre-Mers, Revue d'histoire, $\mathrm{n}^{\circ}$ 376-377, 2OI2, p. I2I-I37.

47. Rapport de la commission chargée d'établir la réforme administrative à Tahiti, 4 décembre I876, ANOM, Fonds ministériels, Séries géographiques, OCEA/35/107.

48. Loi du 30 décembre I 880 dans le Recueil général des lois et des arrêts :en matière civile, criminelle, commerciale et de droit public, Sirey, I88I, p. 86, note 2. Source Gallica.bnf.fr. 
aussi en I88I les Établissements Français d'Océanie (ÉFO) en annexant formellement Tubuaï, Raivaivae et Rapa dans les îles australes ainsi que les Gambier et il s'emploie à organiser de "nouvelles institutions " et en premier lieu un Conseil colonial qu'il veut consultatif. Il prévoit un scrutin à deux listes, Européens et indigènes, six membres européens et six membres indigènes, limité aux seuls territoires de Tahiti et Moorea, pour les premières élections qui se tiennent le 8 août I880. Mais il se heurte à la farouche opposition de la majorité des membres du Conseil d'administration dont certains notables européens de Papeete invités pour l'occasion. Dans la séance du 30 mai I88I, cette majorité réclame la création d'un véritable Conseil général auquel participerait l'ensemble des habitants des archipels, y compris les Marquisiens ou Polynésiens des iles Gambier et des îles australes qui n'ont jamais été liés au Royaume de Pomaré et n'ont donc pas accès à la qualité de citoyen. Il ne faut établir, comme l'avance l'un des participants, aucune distinction entre les intérêts des Océaniens, " craignant [...] de faire naître dans l'esprit des populations exclues un sentiment de jalousie et de défiance à l'égard des seuls Tahitiens. [...] Il n'y a pas lieu de refuser aux populations qui nous entourent l'exercice d'un droit souverain sous le prétexte que quelques-unes de ses fractions sont ou dans l'incapacité ou dans l'impossibilité de le remplir " 49. Le choix d'un scrutin à une liste fait l'objet de longues discussions d'où ressort la préoccupation essentielle de garantir absolument la prédominance des électeurs européens par le jeu du nombre de délégués par circonscription (Papeete étant surreprésentée) ainsi que l'exigence de savoir parler, écrire et lire le français pour les éligibles. Sept circonscriptions sont prévues pour un total de quinze membres élus, six réservés à Papeete, quatre à Tahiti et Moorea, deux aux Tuamotu, un à Tubuaï, un aux Gambier, un à Rapa.

Chessé est résolument opposé à un tel projet: "C'est en même temps qu'une admirable confusion organisée " s'insurge-t-il, " un simulacre de libéralisme qui sous prétexte d'appeler chaque archipel à la gestion des affaires générales des ÉFO ne peut avoir qu'un seul but et un seul résultat celui de décréter au profit de Tahiti et Moorea on peut même dire au profit de Papeete une majorité d'autant plus pernicieuse aux autres archipels qu'elle ne manquera jamais de s'appuyer sur la représentation effective des archipels pour adopter des conclusions absolument contraires aux intérêts de nos archipels " 50.

De son point de vue, comme il l'écrit au ministre des Colonies, « [...] on n'a pas à faire à un pays, mais à un ensemble de pays absolument divers à tous les points de vue. Il suffit de citer les noms de nos archipels, Marquises, Tuamotu, Tahiti, Tubuai, Gambier, pour indiquer

49. Délibération du Conseil d'Administration, 30 mai I88I, ANOM, Fonds ministériels, Séries géographiques, OCEA/ror.

50. Ibid. 
des pays distants entre eux de I00, I50, 200 lieues marines, peuplés de familles (races) différentes, ne parlant pas la même langue ayant des mœurs et des coutumes totalement différentes d'un archipel à l'autre " ${ }^{5}$. Il existe, dira-t-il devant le Conseil d'administration de Tahiti, " une telle différence entre l'état social, les mœurs et les coutumes, la manière de vivre des habitants des divers archipels " à laquelle s'ajoutent "les difficultés de communication, la diversité des besoins et aussi celle des intérêts " qu'il convient d'organiser les Établissements français d'Océanie sous la forme d'une fédération d'archipels ayant chacun leur organisation propre " ${ }^{2}$. Élire des représentants aux Tuamotu lui semble un défi du fait des distances, de l'impossibilité pratique de confectionner les listes électorales alors que l'état civil n'est pas établi, de contrôler les élections, de répondre aux réclamations éventuelles et d'organiser un nouveau scrutin.

"Sur les 80 îles et quelques îles qui composent le groupe des Tuamotu, combien d'ailleurs sont connues, combien ont été visitées par nous, dans les vingt dernières années qui viennent de s'écouler, à quelles difficultés pratiques ne va-t-on pas se heurter dans l'application à leurs habitants des droits et devoirs du citoyen français " 53 .

Et Chessé d'évoquer l'exemple de Temetangui "où il y a eu une affreuse scène de cannibalisme dont a été la victime une famille européenne jetée dans la tempête sur cette île inhospitalière et dont les habitants absolument sauvages, parlant un langage incompréhensible, n'ont pour toute nourriture que du poisson cru, des graines de pandanus et de l'eau saumâtre qu'ils ramassent dans les trous des récifs " 54 . À Tubuai, il n'y a qu'une ferme mal administrée et une population de I2 000 indigènes tandis que Gambier est l'exemple "d'un pays fanatisé par la théocratie ». Ce n'est qu'à Tahiti que l'on peut envisager l'organisation pratique du vote sachant que les 800 Européens que compte l'île sont en majorité à Papeete alors que les $\mathrm{I} 2000$ indigènes se répartissent dans les districts 55. Et encore! Chessé s'emporte, devant le Conseil colonial le 30 juin I88I, contre les effets néfastes de la francisation menée à marche forcée depuis 1842 et l'application mécanique des lois françaises. Il s'en prend en particulier à la période où Roncière appliqua le Code civil et imposa les tribunaux français, sauf dans le domaine foncier (ce qui n'a cependant pas été respecté). Il dénonce avec force les contradictions de la position française qui prétendait " respecter les coutumes tahitiennes" mais qui, dans le même temps,

5I. Lettre du commandant commissaire Chessé du I5 juin I88I, Papeete au Ministre, ANOM, Fonds ministériels, Séries géographiques, OCEA/IOO.

52. Délibération du Conseil d'administration, 30 mai I88I, ANOM, Fonds ministériels, Séries géographiques, OCEA/IOI.

53. Lettre du I5 juillet I88I de Chessé au ministre, ANOM, Fonds ministériels, Séries géographiques, OCEA/IOI.

54. Ibid.

55. Ibid. 
appliquait partout son modèle de droit et de fonctionnement administratif. Sa préoccupation est, au contraire, de "résister " à l'application de la loi française pour un peuple qu'il ne juge pas "mûr " et auquel il faut garantir, sur sa demande même, le respect de ses coutumes ${ }^{56}$.

Chessé dénonce, par ailleurs, les manœuvres des membres du Conseil d'administration qui cherchent à assurer la prépondérance des Européens, "les seuls à même de diriger les débats " 57. L'enjeu sousjacent consiste à obliger tous les archipels à contribuer au budget de la colonie. D'où l'insistance qu'ils soient représentés par un Conseil général élu avec une seule liste, selon un découpage de circonscriptions savamment calculé. Chessé s'insurge contre ces tractations et "demande si le but du Conseil est d'élaborer une constitution pour les établissements de l'Océanie auquel cas c'est à la représentation des intérêts des populations de nos divers archipels qu'il faut pourvoir, ou bien au contraire, s'il n'a en vue qu'une constitution à donner aux trois cents Français (cent cinquante électeurs) auquel cas il faut le dire nettement pour éviter toute confusion" 58 .

Et les conseillers de se draper en réponse dans le respect des principes de la représentation politique pour tous. Le Conseiller Pinaudier exprime avec emphase les enjeux de "la fusion des deux races" :

Il serait tout à fait impolitique de séparer les deux peuples dans l'administration de manière à laisser les Tahitiens faire un corps à part et tout à fait distinct car cela présenterait les plus grands inconvénients, je dirais même de sérieux dangers pour l'avenir. Quand bien même ce mode de procéder n'auraient d'autres inconvénients que d'empêcher la fusion des deux races, je dis qu'à ce seul point de vue, cela serait très regrettable, et qu'il est au contraire d'une bonne politique de chercher à assimiler ces deux races, de les fondre dans une seule, ce qui déjà d'ailleurs a été établi en principe par la loi du 30 décembre 188059 .

L'argument servira à nouveau, mobilisé cette fois par le gouverneur Moreau en I884, lorsqu'il s'agira pour lui de dissoudre le Conseil colonial accusé de "mauvais esprit " et surtout d'utiliser au profit des Européens l'ensemble des recettes tirées de tous les archipels des ÉFO. Moreau impose définitivement une liste unique pour "fusionner les races ", mais dans un conseil redéfini comme général et renouvelé : dix-huit membres dont quatre sont élus à Papeete, six à Tahiti Moorea, quatre aux Tuamotu, deux aux Marquises, un aux Gambier et un à Rapa. Est systématiquement exigée désormais pour les éligibles la connaissance du français, lu, parlé, écrit ${ }^{60}$.

56. Conseil d'administration, séance du 30 juin I88I. Réponse de Chessé, ANOM, Fonds ministériels, Séries géographiques, OCEA/IOI.

57. Délibération du Conseil d'administration I88I. ANOM, Fonds ministériels, Séries géographiques, OCEA/ror.

58. Ibid.

59. Conseil d'Administration, séance du 30 juin I88I, Ibid.

60. Note du gouverneur Moreau, I884, Ibid. 
On connaît peu de choses de la tenue des premières élections et du sens qu'elles ont pu avoir pour les électeurs polynésiens concernés. Le 8 août I880, six Européens sont élus par 320 électeurs européens inscrits (207 votants) et six représentants tahitiens le sont sur leur liste (dont quatre Tahitiens d'origine) et deux d'origine européenne (dont le pasteur protestant Vienot). On ignore quel est le corps électoral tahitien en I880. Ce dernier atteint 2 I 88 électeurs en I 883 et élit trois Tahitiens d'origine et trois Européens sur la liste indigène. Six Européens sont élus alors par 344 votants. Les archipels hors Tahiti et Moorea ne sont pas représentés et ne le seront qu'en I893 et I898 avant d'être à nouveau écartés en I899. Le nouveau Conseil général, en I885, élu sur une liste unique, avec l'obligation de parler, écrire et lire le français, comprend quatorze Européens et un seul Tahitien, le chef Tati Salmon. En 1893, trois Tahitiens représentent les intérêts indigènes, chiffre réduit à deux en $\mathrm{I} 898$ pour un total de dix-huit membres ${ }^{6 I}$.

La citoyenneté active tahitienne et l'exercice du droit de vote en Océanie française, on le voit, n'ont pas grand sens sur le terrain, hormis pour les Français citoyens d'origine européenne. Même pour ces derniers, elle est de courte durée. Entièrement concentrés sur la défense de leurs intérêts, ils entrent dans un conflit persistant avec les représentants de l'administration française, au point qu'en 1903, le gouverneur en vient à demander l'abolition pure et simple du principe de la représentation en Polynésie et de l'existence d'un Conseil général, au profit d'un Conseil colonial fondé sur la nomination de quelques membres de la société civile. Le principe de la citoyenneté active disparaît ainsi dans les Établissements Français d'Océanie et ne sera rétabli qu'à partir de I946. Par une certaine ironie de l'histoire, on pourrait affirmer que le système électif résiste finalement mieux en Nouvelle-Calédonie avec la pérennisation du Conseil général tout au long de la période coloniale, à l'exclusion absolue cependant des Kanak, indigènes non citoyens jusqu'en 1946. Pourtant, le même type d'affrontements s'y joue, entre les représentant des colons au Conseil général, rebelles à toute nouvelle forme d'imposition fiscale et opposés à toute réforme de l'administration visant à améliorer le sort des indigènes. En 1908, le gouverneur Richard propose à son ministre l'élaboration d'un décret en urgence qui supprimerait ni plus ni moins le Conseil général de la Nouvelle-Calédonie pour le remplacer, comme à Tahiti en I903, par un Conseil colonial composé de membres nommés par l'administration ${ }^{62}$. Mais le décret ne voit finalement pas le jour.

Si la citoyenneté dans sa version tahitienne est vidée de son sens en I903, au point qu'un juriste éminent comme Henri Solus semble

6I. Colin Newbury, Tahiti Nui, op. cit., p. 208.

62. Lettre adressée par le gouverneur Richard au ministre des colonies, le 23 décembre 1908. ANOM, Fonds ministériels, IAffpol/266. Voir Isabelle Merle, "De la question de la représentativité d'un peuple "citoyen" en Nouvelle-Calédonie. Retour sur l'histoire", art. cit. 
ignorer quelque vingt ans plus tard que le principe fut un jour appliqué, il convient de noter qu'elle a pourtant eu un effet indéniable. C'est bien ce statut si singulier et éphémère d'" indigène citoyen " qui épargne aux anciens habitants du Royaume de Pomaré, l'imposition officielle du régime de l'indigénat alors que celui-ci devient la norme à partir de I88I pour la très grande majorité des indigènes dans les colonies françaises. Les velléités d'extension de règles démocratiques en Polynésie contribuent, à Tahiti tout au moins, à insuffler un libéralisme colonial avec toute la prudence qu'exige une telle formule, et essentiellement par contraste avec ce qui se joue ailleurs. Les pouvoirs spéciaux dont sont dotés les gouverneurs coloniaux et les règles répressives dérogatoires qui vont avec (internement administratif, séquestre des biens, amendes collectives) sont ici suspendus en I 882 et la liberté de la presse établie ${ }^{63}$. Ce libéralisme, cependant, ne s'étend pas aux îles éloignées comme les Marquises, les Gambier et les îles australes où le statut de "citoyen indigène " n'est que le fruit d'un opportunisme éphémère, ni aux îles Sous-le-Vent où les indigènes sont punis et soumis formellement au régime de l'indigénat jusqu' en $1907^{64}$.

Cependant, à Tahiti comme dans le reste de la Polynésie française, on retiendra surtout l'extrême confusion d'un projet politique français qui semble, dans les années I 880 et de façon très improbable, vouloir expérimenter les règles électives sur les marges les plus lointaines de son empire. Le projet est en soi surprenant et il était intéressant de le rappeler tant il a été oublié, y compris par les Tahitiens eux-mêmes, pour lesquels l'exercice de la citoyenneté n'a de sens que depuis I946. Cet oubli n'a rien d'étonnant, car les velléités démocratiques de la France ont été de courte durée, remplacées dès 1903 par la formule classique de l'exercice du pouvoir administratif en contexte colonial. L'érosion inéluctable des droits de citoyens accordés en I880 aux anciens sujets du Royaume de Pomaré, les conduit finalement sur la voie d'une indigénisation progressive de leur statut. Certes, ils échappent à l'application du régime de l'indigénat, mais c'est sans doute davantage parce que celui-ci s'avère inutile pour contrôler une population qui reste très encadrée dans les district par les règles religieuses protestantes et les codes de lois hérités des évangélistes britanniques et dont le respect est garanti par les chefferies et la police locale des mutoï. ${ }^{5}$ Les gouverneurs n'exercent pas de pouvoirs spéciaux à Tahiti, mais ils ont tout pouvoir pour le faire dans les territoires périphériques, aux Marquises, aux Gambier ou aux îles Australes et sur les îles Sous-le-Vent. Les indigènes polynésiens, par ailleurs, sont soumis aux impôts dits de

63. Sur la question des pouvoirs spéciaux, voir Isabelle et Adrian Muckle, op. cit. et Sylvie Thénault, Violence ordinaire dans l'Algérie coloniale. Camps, internements, assignations à résidence, Paris, Odile Jacob, 2012.

64. Sur le cas particulier des îles Sous-le-Vent, voir Isabelle Merle et Adrian Muckle, L'indigénat, op. cit., p. II8-II 9.

65. Nom tahitien donné à la police des chefferies. 
route ou aux travaux obligatoires et autres prestations. Au début du $\mathrm{xx}^{\mathrm{e}}$ siècle, une même condition indigène est partagée finalement par l'ensemble des habitants des Établissements d'Océanie. Mais celle-ci diverge indéniablement de celle que vivaient au même moment les indigènes de Nouvelle-Calédonie. Alors que nombre de Polynésiens résident dans les districts à l'abri des chefferies et des paroisses, relativement éloignés de l'emprise coloniale, les Kanak, quoique refoulés dans les réserves, subissent, via l'indigénat, une exploitation de travail et une répression quotidienne sans commune mesure.

Les promesses de la loi de $\mathrm{I} 880$ à Tahiti ont donc fait long feu mais elles ont eu au moins une conséquence de taille en épargnant aux anciens sujets de Pomaré l'imposition du régime de l'indigénat qui a accompagné, tout au long de la période coloniale, le statut des indigènes non citoyens français. 